\section{Molecular oncology}

\author{
Antony J. Michalski
}

Genes and Cancer. Edited by Desmond Carney and Karol Sikora. Wiley: 1990. Pp. 348. £24.95, \$49.50.

Molecular Genetics and Cancer Dlagnosis. By Jeffrey Crossman. Elsevier: 1990. Pp. 465. Dfl165, \$69.50.

THE last two decades have seen an exponential rise in the amount and diversity of research in molecular biology. More recently, results of this technology have made an impact on clinical practice. Recombinant DNA techniques are used to produce growth hormone, insulin and haemopoietic growth factors. Identification of relevant restrictionfragment length polymorphisms is now routine in carrier detection and prenatal diagnosis of genetic diseases. The polymerase chain reaction is being used to amplify virusspecific sequences in the diagnosis of various virus infections, for example, human immunodeficiency virus in neonates. The genetic events responsible for tumorigenesis in retinoblastoma and colon cancer have now been identified and huge steps are being made in understanding the molecular pathogenesis of Wilms' tumour, Burkitts lymphoma and many other malignancies. No longer can clinicians and 'nonmolecular' scientists remain ignorant of the basic principles, techniques and jargon of molecular biology and they need a succinct and understandable introduction to this highly complex field. Genes and Cancer edited by Desmond Carney and Karol Sikora, and Molecular Genetics and Cancer Diagnosis by Jeffrey Crossman are aimed at a general readership and attempt to fulfil this role, though in different ways.

Genes and Cancer is divided into six sections covering oncogenes, gene control, tumour biology, growth factors and receptors, drug resistance and the use of antibodies for cancer therapy. The sections contain between four and eight articles of around ten pages in length and are followed by short but well-chosen lists of further reading. The authorship is largely UK based but there are contributions from the United States and Japan. The strength of the book is its broad scope and the many chapters that provide excellent reviews of a topic with the minimum of extraneous detail. In any evolving field, deciding on the balance between communicating hard data and speculative interpretation is difficult, but some chapters are made less digestible for a general readership by the inclusion of the full sequence of an oncogene or the configuration of its protein product. It is impossible for a book of this size to be comprehensive but I was surprised not to find a single chapter on 'tumoursuppressor' genes. Similarly, although paediatric malignancy numerically accounts for a tiny proportion of all cancer, it could have received more space if only to use retinoblastoma as a vehicle to discuss tumour-suppressor genes or Wilms' tumour to discuss genomic imprinting.

By contrast Molecular Genetics in Cancer Diagnosis is narrower in scope but more cohesive, with themes progressively developed by a variety of authors. It is divided into 25 chapters with up to 12 subheadings in each. The authorship is virtually all North American and has chosen to give comprehensive, but rather daunting, reading lists at the end of each chapter. The book concentrates much more on the application of formal molecular biology to oncology and does not attempt to cover current or future treatment modalities in any detail. The first eight chapters form a well-written, succinct introduction to the genetic basic of cancer, molecular techniques, oncogenes, tumour-suppressor genes, growth factors, metastasis and drug

\section{Awaiting tests}

\section{Neil Turok}

\section{Particle Physics and Inflationary Cosmology. By Andrei D. Linde. Harwood: 1990. Pp.362. £32, \$60.}

THE idea that one or more phase transitions occurred in the early Universe lies behind most of the theoretical work in cosmology over the last two decades. Particle Physics and Inflationary Cosmology is one of the first books devoted to a detailed technical treatment. Its author has been intimately involved in the subject since its inception, and has consistently been one of the most active and imaginative workers in the field. In the early 1970 s, Andrei Linde was one of the first to realize that the standard electroweak theory predicts such a phase transition, and in the early 1980 s played a leading role developing the inflationary model, through which the dynamics of a grand unified phase transition could resolve many of the basic conundrums of cosmology.

Linde has since then pursued new models of inflation, and their consequences, with great vigour. His particular strength is in finding simple physical arguments to clarify and explain the very complex processes involved. This comes through strongly in his book, which provides simple but quite deep treatments of many of the more technical issues - effective potentials, spontaneous symmetry breaking, the generation of fluctuations. It should serve as both a textbook and a standard reference on inflation for years to come. It is packed with original and intuitive viewpoints on finite-temperature field theory, inflation and quantum gravity. The emphasis is always on simple physical pictures and rough scaling arguments rather than on rigorous mathematical formalism. This can be a little frustrating - for example in the resistance. The chapter on the cytogenetic basis for molecular analysis of malignancies is particularly useful. The remaining chapters discuss the molecular pathology of selected malignancies. The majority are 'disease based' explaining the relevance of cytogenetic abnormalities, oncogene activation and loss of tumour-suppressor gene function to the tumour under discussion. There are several chapters on haematological malignancies but the solid tumours receive less complete coverage in terms of the variety of diagnoses considered, though the articles themselves are amongst the best in the book. Although some of the chapters are more detailed than others, and a few communicate data in a more philatelic than interpretative way, this is a readable and informative book.

Antony J. Michalski is in the Department of Haematology and Oncology, Institute of Child Health, 30 Guilford Street, London WC1N $1 E H, U K$.

discussion of quantum gravitational effects, for which there is as yet no consistent theory, but which are treated in a crude intuitive manner. But Linde's point of view is always simple and stimulating.

The first half of the book is a nice review of phase transitions in field theories, including those in the standard model, bubble nucleation and topological defects. The main emphasis in the second half of the book is on an enthusiastic account of the many versions of inflation and in particular of two models: 'new inflation' and 'chaotic inflation'. The latter has come to be seen as probably the simplest embodiment of the inflationary hypothesis. But chaotic inflation is still on rather shaky footing: the initial conditions 'before' it are discussed using very rough arguments close to the Planck era, where unknown quantum gravity effects are undoubtedly important. This vagueness lends a note of unreality to the discussion. The book closes with a long heuristic discussion of quantum cosmology, the 'self-regenerating universe' which is a development of the chaotic model, and the anthropic principle. Somewhat disappointingly it thus ends firmly in the realm of philosophy rather than experiment.

My main criticism of the book is that it contains barely a mention of the observational data. Linde's primary concern is with the basic aesthetic problems of the hot Big Bang, and their resolution in theories of inflation, for which he is a very strong and able proponent. Inflation provides a simple dynamical mechanism through which a small lumpy 'typical' universe could become large, flat and smooth like our own. At present there is no other mechanism known which does this, barring the supposition of special initial conditions, which may in the end always resolve these issues. Furthermore, inflation provides a mechanism for the generation of small-amplitude primordial 'wrinkles' on the universe, which later grow 